\title{
Generalized network theory of physical two-dimensional systems
}

\author{
David Ormrod Morley $\odot,{ }^{1}$ Alice L. Thorneywork, ${ }^{1,2}$ Roel P. A. Dullens, ${ }^{1}$ and Mark Wilson $\oplus^{1, *}$ \\ ${ }^{1}$ Department of Chemistry, Physical and Theoretical Chemistry Laboratory, University of Oxford, Oxford OX1 3QZ, United Kingdom \\ ${ }^{2}$ Cavendish Laboratory, University of Cambridge, Cambridge CB3 OHE, United Kingdom
}

(Received 12 August 2019; revised manuscript received 22 January 2020; accepted 26 March 2020; published 27 April 2020)

\begin{abstract}
The properties of a wide range of two-dimensional network materials are investigated by developing a generalized network theory. The methods developed are shown to be applicable to a wide range of systems generated from both computation and experiment; incorporating atomistic materials, foams, fullerenes, colloidal monolayers, and geopolitical regions. The ring structure in physical networks is described in terms of the node degree distribution and the assortativity. These quantities are linked to previous empirical measures such as Lemaitre's law and the Aboav-Weaire law. The effect on these network properties is explored by systematically changing the coordination environments, topologies, and underlying potential model of the physical system.
\end{abstract}

DOI: 10.1103/PhysRevE.101.042309

\section{INTRODUCTION}

Two-dimensional (2D) networks are ubiquitous in the natural world, emerging across all physical disciplines and length scales. Traditional examples range from the atomic level of ultrathin materials [1-3], through colloids [4,5], foams [6,7], epithelial cells [8,9], and geological rock formations (see Fig. 1) [10]. There are, however, countless more occurrences, with drying blood [11], stratocumulus clouds [12], and geopolitical borders [13] all being the subject of studies. Although these systems are incredibly physically diverse, it has long been noted that they have strikingly similar properties [14]. This is because they can all be mapped onto a generic system, which can be equivalently described as a collection of tessellating polygons or percolating rings, and hence they are governed by the same fundamental laws. Understanding the behaviours of two-dimensional networks is therefore key to a wide range of problems in cutting-edge research, including the control of mitotic division $[15,16]$ and directed synthesis of nanomaterials $[17,18]$, as well as to curiosities such as explaining the arrangement of the stones in Giant's Causeway [19] or cracking in famous artworks [20].

A consequence of this universality is that both the language and the metrics used to describe two-dimensional networks varies considerably between fields, as demonstrated in Table I. From a nanomaterials perspective we may think of rings being formed from a set of bonded atoms, in crystals we have grains separated by boundaries and in biological tissues cells which divide. Further complication may arise from the concept of graph duality, where ring structure emerges only after transforming the physical coordinates. In the context of colloidal monolayers, for instance, rings are generated using the Voronoi construction, where the vertices have no real manifestation and the particle positions are the simplices in the dual Delaunay triangulation. However, the continuing expansion and maturity of network science as a field has

\footnotetext{
*mark.wilson@chem.ox.ac.uk
}

led to new ways of thinking about and quantifying complex networks [21]. These advances were largely driven by interest in networks in the more abstract sense of the internet, social media, and neural networks [22,23], with applications to physical systems still being more confined to topics such as biological signaling pathways.

In this paper we therefore aim to apply the techniques of network science to the analysis of commonly occurring physical systems to demonstrate how better to quantify their structure. As part of this process we will recast and extend some of the existing empirical observations of physical networks, namely Lemaitre's law and the Aboav-Weaire law, on the distributions and arrangements of rings. We will also develop both simulation and experimental methods to generate realizations of complex two-dimensional networks, comprising a variety of computational Monte Carlo techniques as well as production of colloidal monolayers. We will analyze these configurations alongside existing available experimental coordinates from two-dimensional materials $[1,2,24]$ to explore the properties of a range of commonly studied systems encompassing materials, foams, and colloids.

\section{THEORY}

\section{A. Network description}

All the physical networks considered in this work can all be viewed as a collection of linked nodes, where the entities that the nodes and links represent depend on the system in question. For example, in materials they could represent atoms and bonds or in colloids the vertices and edges of Voronoi cells. The networks are also planar, in the sense that no links overlap. An example of this network is given by the square nodes in Fig. 2. The number of links to each node in the network is termed the node degree, which we will denote $c$. In atomic systems, this is termed the coordination number, i.e., the number of atoms each atom is bonded to. For the vast majority of natural random networks, including the Voronoi diagram, all node degrees are equal to three, as higher-order vertices are unstable with respect to even the 
TABLE I. Terminology to describe ring structure in literature reflects the diversity of the underlying physical systems.

\begin{tabular}{lc}
\hline \hline Term & Synonyms and Examples \\
\hline Ring & Face, polygon, cell, grain, pore, Voronoi cell \\
Network & $\begin{array}{c}\text { Graph, tiling, packing, tessellation, partition, } \\
\text { arrangement, decomposition, net, mosaic }\end{array}$ \\
Link & Edge, bond, boundary, interface \\
Node & Vertex, point, atom \\
\hline \hline
\end{tabular}

smallest perturbations and readily split into multiple lowerorder sites [25]. The main exception is in atomic systems, where the exact number of bonds depends on the chemistry of the elemental species involved. Therefore while this network is often the most physically intuitive, it does not capture the disorder of the system well, as in the first instance all nodes appear equivalent.

Instead, we note that the system can also be viewed as percolating rings of different sizes, where the size of a ring, $k$, is defined by the number of its constituent nodes. From this we may construct a dual network, where a node is placed at the center of each ring and the nodes of adjacent (i.e., edgesharing) rings linked. In our colloidal systems, for example, this would be equivalent to the Delaunay triangulation. An example of this network is given by the circular nodes in Fig. 2. The node degree in this dual network, $k$, is then equal to the ring size in the original network. In addition, the links now describe the ring adjacencies. The direct relationships between the physical descriptions and network measures are given in Table II and discussed in detail below.

\section{B. Average degree}

An interesting consequence of the well-defined local coordination environments in physical systems is that the average node degree of the ring network, $\langle k\rangle$, becomes constrained. As the networks considered in this work are planar, they are subject to Euler's formula given as:

$$
N+V-E=\chi,
$$

where $N, V, E$ are the number of rings, vertices, and edges in the graph and $\chi$ is an integer termed the Euler characteristic, which is topology dependent. A generic physical network consists of $c$-degree vertices, with the proportion of each type given by $x_{c}$, where $\sum_{c} x_{c}=1$, and the mean node degree is therefore $\langle c\rangle=\sum_{c} c x_{c}$. This allows the number of edges to be written in terms of the vertices as $E=\frac{V}{2}\langle c\rangle$. In turn the mean ring size is simply the total number of vertices per ring, allowing for multiple counting, so that $\langle k\rangle=\frac{V}{N}\langle c\rangle$. Substituting these two expressions into Eq. (1) leads to the expression:

$$
\langle k\rangle=\frac{2\langle c\rangle(1-\chi / N)}{\langle c\rangle-2} .
$$

Hence we find that the average node degree in the ring network (equivalent to the mean ring size of the physical network) is simply related to the average degree of the physical network (i.e., local coordination environment), the topology of the system, and the number of rings.

Although Eq. (2) may appear simple, it is a very powerful constraint. To demonstrate this consider a two-dimensional lattice with two possible coordination environments $c=3,4$. The planar case with periodic boundary conditions maps onto the torus with $\chi=0$, and so we find:

$$
\langle k\rangle=\left\{\begin{array}{ll}
6, & x_{3}=1 \\
4, & x_{4}=1 \\
5, & x_{3}=2 / 3, x_{4}=1 / 3
\end{array} .\right.
$$

To reiterate in plain terms, this means that if we have a material consisting of atoms all forming exactly three bonds (as (a)

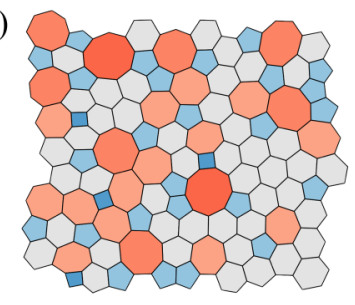

(e)

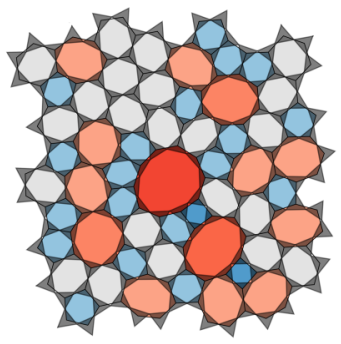

(b)

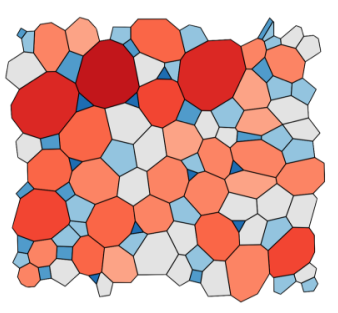

(f)

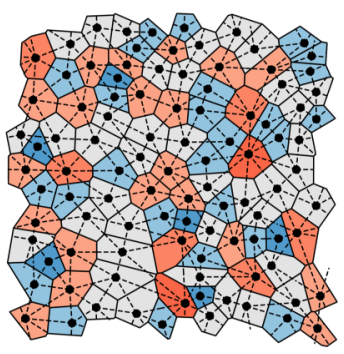

(c)

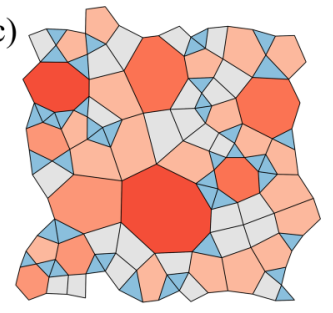

(d)
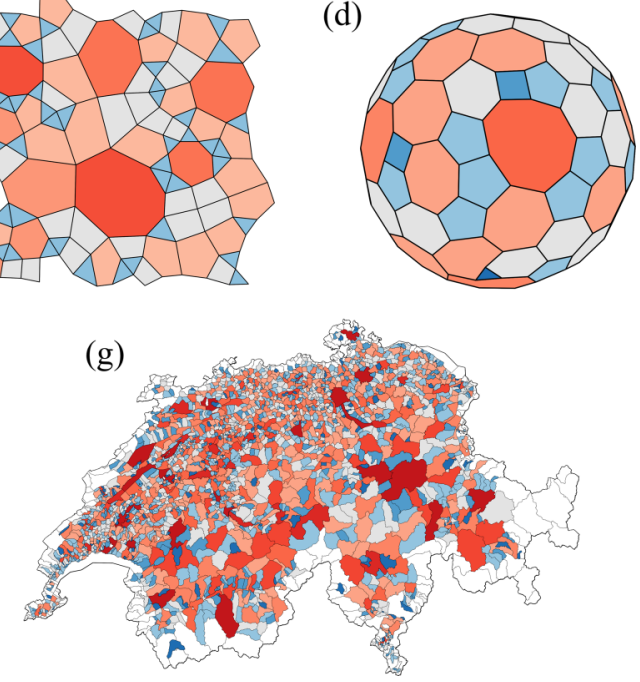

FIG. 1. Two-dimensional networks emerge in diverse physical systems and span a range of length scales, coordination environments, and topologies: (a) 3-coordinate, bond-length controlled network, e.g., glass; (b) 3-coordinate, angle controlled network, e.g., foam; (c) 4coordinate network; (d) 3-coordinate network in spherical geometry, e.g., nonclassical fullerene; (e) triangle raft, e.g., silica bilayer; (f) Voronoi partition, with dual network highlighted (black points and dashed lines), e.g., colloidal monolayer; and (g) communes of Switzerland. Rings are colored similarly according to size with blue, gray, and red indicating smaller than, equal to, and greater than the mean ring size, respectively. 


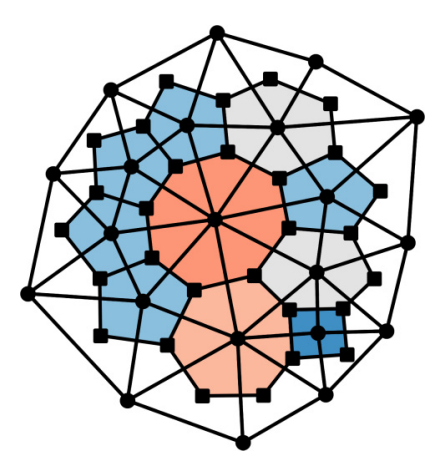

FIG. 2. An example physical network is given by the linked square nodes, which all have node degree (or coordination) of three. The dual network is given by the linked circular nodes, which have a node degree equal to the ring size in the physical network. This network therefore describes the ring structure.

for amorphous carbon), then the mean ring size must be equal to 6 . For aperiodic systems strictly $\chi=1$, but as $N$ grows and the proportion of vertices with unsatisfied coordination on the sample perimeter become negligible overall, in reality these relationships hold and remain as applicable to amorphous graphene [26] as the basalt columns in Fingal's cave [10] and the Penrose tiling [27].

This analysis extends to spherical topology where $\chi=2$, and so:

$$
\langle k\rangle=\left\{\begin{array}{lc}
\frac{6 N-12}{N}, & x_{3}=1 \\
\frac{4 N-8}{N}, & x_{4}=1
\end{array} .\right.
$$

These relationships are the origin of the 12-pentagon rule for 3-coordinate fullerenes [28] (the "football problem"), or equivalently an "8 triangle rule" in the 4-coordinate case, as this is the only way to satisfy these equations if the allowed ring sizes are limited to $k=5,6$ and $k=3,4$, respectively. Much of the richness in the behavior stems from this fundamental constraint on the network average degree.

\section{Degree distribution}

Knowing that the mean node degree is fixed, the next level of available information is the form of the underlying degree distribution, $p_{k}$. In materials science this quantity is often referred to as the ring statistics and encodes information about the level of disorder in an amorphous system [29]. Perhaps surprisingly, the degree distributions found in physical ring networks seem relatively well defined. It was noted in silica that the ring statistics looked to follow a lognormal distribution [30], but Lemaître et al. demonstrated that the distribution

TABLE II. Network measures directly correspond to traditional quantities of ring systems.

\begin{tabular}{lcc}
\hline \hline Symbol & Network measure & Physical description \\
\hline$k$ & Degree & Ring size \\
$p_{k}$ & Degree distribution & Ring statistics \\
$e_{j k}$ & Joint degree distribution & Ring connectivity \\
$r$ & Assortativity & Ring correlations \\
\hline \hline
\end{tabular}

in 3-coordinate networks systems can be described by a maximum entropy distribution [31]. We summarize the maximum entropy method here, noting that there is a trivial extension to arbitrary coordination.

The entropy of a probability distribution is defined as $S=-\sum_{k} p_{k} \log p_{k}$. The degree distribution has the following constraints:

$$
\begin{aligned}
\sum_{k} p_{k} & =1, \\
\sum_{k} k p_{k} & =\langle k\rangle, \\
\sum_{k} p_{k} / k & =\text { const, }
\end{aligned}
$$

where the first two constraints correspond to the normalization condition and the fixed mean ring size. The final constraint will be discussed below. The entropy can then be maximized using Lagrange's method to yield the result:

$$
p_{k}=\frac{e^{-\lambda_{1} k-\lambda_{2} / k}}{\sum_{k} e^{-\lambda_{1} k-\lambda_{2} / k}},
$$

which can be solved numerically by substitution into Eqs. (6) and (7). By allowing the chosen constant to vary, a family of maximum entropy curves can be generated. The resulting distributions can be summarized by relating the variance, $\mu_{2}=\left\langle k^{2}\right\rangle-\langle k\rangle^{2}$, to a single chosen node degree probability, leading to the plot known as Lemaitre's law. It is usually framed in the context of the proportion of hexagons in a system, $p_{6}$, for the precise reason that most networks have $\langle k\rangle=6$ and $p_{6}$ as the largest contribution. Many experimental and theoretical studies have shown good agreement to this law $[25,32,33]$. Simple extensions of the classic law are, however, possible by modifying the mean degree or the permitted degree range. We note that $k$ is usually taken in the interval $k \geqslant 3$ (as the triangle, $k=3$, is the smallest polygon), but there could in principle be manifestations of physical systems where only certain degrees are accessible.

The only somewhat puzzling aspect of this successful theory is the choice of constraint (7). It was originally rationalized on the basis that the areas of rings of a given size, $A_{k}$, can be well fit by an expression $A_{k}=a k+b+c / k$, where $a, b$, and $c$ are constants. As noted at the time, this is by no means true for all systems [34] and in fact is contrary to the widely known Lewis law [35], which states that $A_{k}$ is linear in $k$ for many observable networks [36-38]. Despite this, the universality of the Lemaitre law suggests that there must be a physical basis to (7), and in the following section we demonstrate that it can be regenerated by considering ring adjacencies.

\section{Assortativity}

The degree distribution is a very important measure for physical networks, but it does not provide a complete characterization of the ring structure, as it says nothing about the ring adjacencies. This is important because while with the same ring statistics it is theoretically possible to organize the rings in many different arrangements, it is well known experimentally that only a subsection of these are observed. In 
fact, the vast majority of physical systems have a preference for small rings $(k<\langle k\rangle)$ be adjacent to large rings $(k>\langle k\rangle)$.

Traditionally, in physical sciences the extent of this smalllarge ring pairing is quantified through the empirical AboavWeaire law [38-40],

$$
k m_{k}=\langle k\rangle^{2}+\mu_{2}+\langle k\rangle(1-\alpha)(k-\langle k\rangle),
$$

where $m_{k}$ is the mean ring size about a ring of size $k$ and $\alpha$ is a fitting parameter. The value of $\alpha$ describes the ring correlations, with a larger positive value indicating a greater tendency for small-large ring adjacencies. The law is well used, for example, in studies of materials [41,42], emulsions [43], biological tissues [44], as well as in planetary science [45].

There are, however, limitations with quantifying ring correlations in this way. First, it is not intuitive what the actual value of $\alpha$ or its limits represent. It can be shown by evaluating $\frac{\partial m_{k}}{\partial k}=0$ that if rings were arranged purely randomly (the "topological gas" [31]) then $\alpha=-\mu_{2} /\langle k\rangle^{2}$. This highlights both that $\alpha$ is dependent on the ring statistics and that its sign is an insufficient classifier. Second, and more critically, the assumption Aboav-Weaire law holds and is indeed linear is often overlooked $[4,46,47]$. The determination of the $\alpha$ parameter therefore always has a degree of grayness. A combination of these effects make it difficult to draw accurate comparisons between different systems.

However, the analogous problem in network science is already well established. The assortativity was introduced by Newman to measure the preference of low-degree nodes to be adjacent to high-degree nodes in generic networks [48]. It has proved highly popular in the network science and the study of social and biological networks [49] but has also been applied for example in theoretical studies of hard-disk packings [50]. The calculation of the assortativity revolves around the edge joint degree distribution, $e_{j k}$, which measures the probability of two nodes of degrees $j, k$ sharing a link (i.e., two rings of sizes $j, k$ being adjacent). The probability of any link having degree $k$ is distributed according to $q_{k}=k p_{k} /\langle k\rangle$, and so if nodes are randomly arranged, then $e_{j k}=q_{j} q_{k}$. Deviation from this random arrangement is the assortativity and can be measured by Pearson's correlation coefficient:

$$
r=\frac{\sum_{j k} j k\left(e_{j k}-q_{j} q_{k}\right)}{\sum_{k} k^{2} q_{k}-\left(\sum_{k} k q_{k}\right)^{2}}=\frac{\langle k\rangle^{2} \sum_{j k} j k e_{j k}-\left\langle k^{2}\right\rangle^{2}}{\langle k\rangle\left\langle k^{3}\right\rangle-\left\langle k^{2}\right\rangle^{2}} .
$$

For this coefficient to be calculable, the second and third moments of the degree distribution must be finite [51]. This condition is satisfied for these physical systems, as the proportion of large rings quickly becomes vanishingly small.

The advantages of adopting this measure of assortativity are clear. The correlation coefficient is bounded between $-1 \leqslant r \leqslant 1$ and has three well-defined limits: $r=0$, indicating a random network; $r=1$, a perfectly assortative network; and $r=-1$, a perfectly disassortative network. This allows physical networks to be readily compared in a way that the Aboav-Weaire law does not allow. Physical networks can now be fitted in to the wider field of network science, introducing them as important examples alongside more traditionally studied networks. Using the assortativity also provides a natural extension to higher dimensions, which has been difficult to reconcile with the empirical Aboav-Weaire law [52].

For completeness, we will, however, show that the assortativity can be related to the Aboav-Weaire parameter. To achieve this we use the fact that that the mean node degree about a $j$-degree node can be expressed $\sum_{k} k e_{j k}=q_{j} m_{j}$. Substituting this expression into Eq. (10), and assuming the Aboav-Weaire law (9) holds exactly, it can be shown that:

$$
\alpha=-\frac{r\left(\langle k\rangle\left\langle k^{3}\right\rangle-\left\langle k^{2}\right\rangle^{2}\right)}{\mu_{2}\langle k\rangle^{2}}-\frac{\mu_{2}}{\langle k\rangle^{2}}
$$

which is consistent with the topological gas, when $r=0$. In reality, the Aboav-Weaire fit is never perfect, and so Eq. (11) provides an approximation to the value of $\alpha$. The accuracy of this equation will therefore depend on the applicability of the linear fit.

The assortativity also provides a natural framework to extend Lemaître's maximum entropy arguments to factor in ring adjacencies. We now define the entropy in terms of the edge joint degree distribution, as $S=-\sum_{j k} e_{j k} \log e_{j k}$. Considering $e_{j k}$, the following constraints must hold:

$$
\begin{gathered}
\sum_{j k} e_{j k}=1, \\
\sum_{j k} k e_{j k}=\frac{\mu_{2}}{\langle k\rangle}+\langle k\rangle, \\
\sum_{j k} \frac{1}{j} e_{j k}=\frac{1}{\langle k\rangle}, \\
\sum_{j k} j k e_{j k}=c(r),
\end{gathered}
$$

resulting from the normalization condition, Weaire's sum rule [40], and Euler's formula and finally a constraint imposing the assortativity from Eq. (10). As for Lemaître's law, Lagrange's method can be used with the constraints above (noting that $e_{j k}=e_{k j}$ ) to generate a maximum entropy joint distribution which satisfies:

$$
e_{j k}=\frac{e^{-\frac{\lambda_{1}}{2}(j+k)-\frac{\lambda_{2}}{2}(1 / j+1 / k)-\lambda_{3} j k}}{\sum_{j k} e^{-\frac{\lambda_{1}}{2}(j+k)-\frac{\lambda_{2}}{2}(1 / j+1 / k)-\lambda_{3} j k}}
$$

and Eqs. (13)-(15). This can again be solved numerically, and the resulting distribution can be related to a single node degree probability (e.g., $p_{6}$ ) and an assortativity value.

\section{COMPUTATIONAL METHODS}

Here we discuss different ways to generate configurations for network analysis. The primary method we will use in this work is the bond switching algorithm, due to its flexibility, but results will also be supplemented with data generated using the triangle raft algorithm and hard-disk Monte Carlo. 
TABLE III. List of starting crystalline lattices for bond switching for a range of coordinations.

\begin{tabular}{lccc}
\hline \hline Topology & $x_{3}$ & $x_{4}$ & Lattice \\
\hline Planar & 1 & 0 & Hexagonal \\
Planar & 0 & 1 & Square \\
Planar & $2 / 3$ & $1 / 3$ & Cairo \\
Planar & $x_{3}$ & $x_{4}$ & Mixed hexagonal-square \\
Spherical & 1 & 0 & 12-Pentagon fullerene \\
Spherical & 0 & 1 & 8-Triangle fullerene \\
\hline \hline
\end{tabular}

\section{A. Bond switching}

The bond switching algorithm is a Monte Carlo approach for generating periodic disordered network configurations which are well relaxed. It was originally developed in three dimensions by Wooten, Winer, and Weaire to generate highquality models of silica glass [53]. Analogous approaches have since been utilized for 3-coordinate, and in one instance 4-coordinate, planar atomic systems [54,55]. We present a further natural extension of the bond switching method to variable atomic coordination environments and overall system topology. As a review from a networks perspective, the bond switching algorithm is a stochastic sampling method. Starting from an initially well-ordered network, links between neighboring nodes are switched and the effect on the potential energy of the system (i.e., the amount of strain which is introduced or removed) is calculated. The energy of the system is determined by the potential model, which expresses the total energy of the network as a function of all node positions. After the links between nodes are switched, geometry optimization of the node positions takes place to minimize the total potential energy. By incorporating switches which reduce the potential energy of the network with greater probability, one can bias the search towards networks of lower energy and therefore which occur more commonly in nature.

The general algorithmic procedure for bond switching is as follows:

(1) Generate initial crystalline atomic lattice i.e., make a regular array of nodes with known degrees.

(2) Thermalize the lattice with a large number of random moves, i.e., switch links between nodes to generate a random network with high energy.

(3) Anneal the system at finite temperature, $T$, by accepting moves according to the Metropolis criterion [56]:

$$
P=\min \left[1, e^{-\Delta U / k_{B} T}\right],
$$

where $\Delta U$ is the energy change as a result of the proposed move, i.e., continue to switch links in the network, but now accepting changes which reduce energy with greater probability.

The specificities of the algorithm will, however, depend on the exact nature of the system in question. In particular, the choice of the starting lattice can be used to determine the system properties in terms of the atomic coordination environments and topologies (Table III, Fig. 3). This is because in the bond switching algorithm the node degree distribution of the atomic network is constant, and hence from Eq. (2) so is the mean node degree of the dual network.

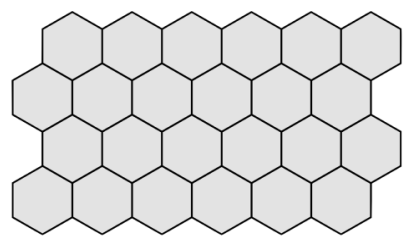

(a)

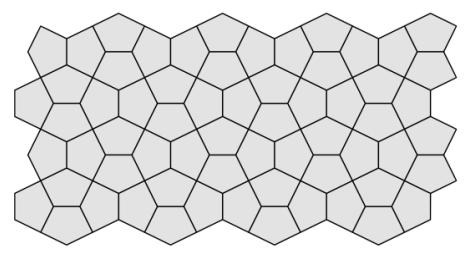

(c)

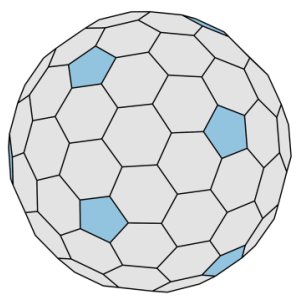

(e)

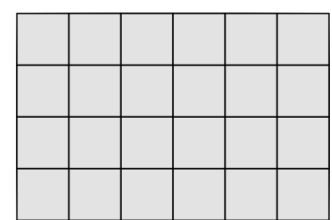

(b)

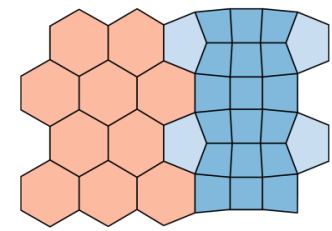

(d)

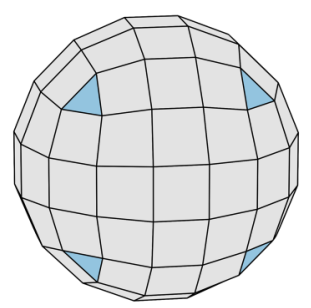

(f)
FIG. 3. The choice of starting lattice can be used to select coordination properties, and therefore $\langle k\rangle$, in the bond switching algorithm. (a) Hexagonal net, (b) square net, (c) Cairo lattice, (d) mixed threeand four-coordinate lattice, and [(e) and (f)] fullerenes based on hexagonal and square nets, respectively.

The bond switching move will then vary depending on the coordination properties, as outlined in Fig. 4. The first [Figs. 4(a)-4(c)] is the original move, which was designed for purely 3-coordinate atoms, and is in effect introducing a Stone-Wales defect. We note that this move augments the ring size of two rings and decrements two others, preserving both the mean ring size and the coordination number of the individual atoms involved in the transformation. The changes in ring size (equivalent to the changes in node degree of the dual network) are highlighted in the figure as " \pm 1 ." The extension to 4-coordinate atoms [Figs. 4(d)-4(f)] is relatively straightforward, simply involving extra spectator atoms, but for mixed coordination it is subtly different [Figs. 4(g)-4(i)]. For both systems the local ring sizes are again changed by \pm 1 (as highlighted and preserving the mean ring size). However, whereas for the pure systems the switch move must be coordination preserving, for mixed coordination systems this prevents true melting. This can be countered by using a move in which the coordinations of neighboring atoms are exchanged while maintaining a constant mean ring size. As the effects of the bond switching moves on the ring network are clearly well defined, this also opens the possibility of manipulating the ring network directly, while maintaining the rules of the atomic space $[57,58]$.

The thermalization of the initial lattice requires a large number of random moves as described above, the purpose being for the system to "forget" all memory of the original ordered lattice. To ensure the lattice is fully randomized, 


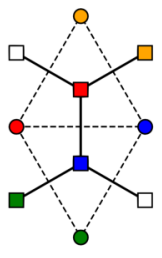

(a)

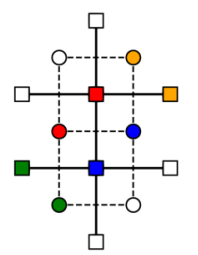

(d)

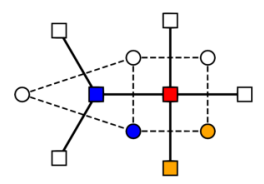

(g)

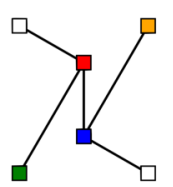

(b)

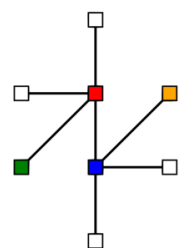

(e)

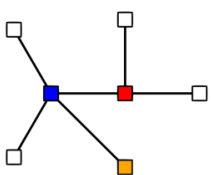

(h)

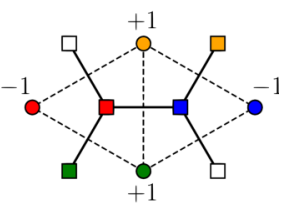

(c)

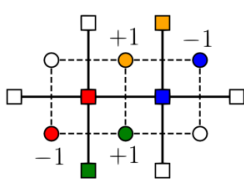

(f)

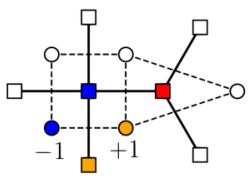

(i)
FIG. 4. Bond switching Monte Carlo moves differ depending on atomic coordination environment, as shown for 3-coordinate sites [(a)-(c)], 4-coordinate sites [(d)-(f)], and mixed 3/4 coordination [(g)-(i)]. For each coordination type the atomic connectivity is shown for the starting structure (left), the initial switched structure (middle), and a geometry optimized switched structure (right) via the squares and solid lines. The effect on the dual network (circles and dashed lines) is also demonstrated, with the numbers indicating the change in node degree after the move is applied. Coloring is used as a guide for the eye to track changes between the pre- and postswitch configurations.

observables such as the second moment of the ring sizes and assortativity can be monitored. For mixed lattices it is also important that the variously coordinated atoms are adjacent to the number of others as expected from pure chance, namely the binomial expansion of $\left(3 x_{3} /\langle k\rangle+4 x_{4} /\langle k\rangle\right)^{2}$.

A key aspect in the bond switching algorithm is the potential model, which is required for geometry optimization after the transposition and evaluation of the system energy. The method naturally lends itself to the use of semiempirical potentials which have explicit bonding and angular neighbor lists, and as such a popular choice is the Keating potential $[59,60]$ or modifications thereof [61]. For this work we have also opted to use a simplified two-dimensional version of the Keating potential [62] with the option of being augmented with a restricted bending $(\mathrm{ReB})$ potential [63]. This has the form:

$$
\begin{aligned}
U= & \sum_{i, j \in \text { bonds }} \frac{k_{r}\left(r_{i j}-r_{0}\right)^{2}}{2} \\
& +\sum_{i, j, k \in \text { angles }} \frac{k_{\theta}\left(\cos \theta_{i j k}-\cos \theta_{0}\right)^{2}}{f\left(\theta_{i j k}\right)},
\end{aligned}
$$

where $r_{0}$ is the equilibrium separation between atoms and $\theta_{0}$ the equilibrium angle and $k_{r}, k_{\theta}$ are the respective bond and angle force constants. The equilibrium bond length was set equal for all interaction types and the equilibrium angles were set to $2 \pi / c$ for $c$-coordinate atoms. The function $f\left(\theta_{i j k}\right)$ can either be set to unity or $f\left(\theta_{i j k}\right)=2 \sin ^{2} \theta_{i j k}$ to obtain the simplified Keating (SK) and ReB potentials, respectively. In addition, to generate networks in spherical geometry, a simple harmonic restraining potential was added to all atoms to keep them on a sphere of a fixed radius.

The rational for choosing this potential is that we aim to obtain results for generic systems, generating configurations with a high-throughput approach. This model captures the essential physics the problem while remaining computationally tractable when producing a large number of samples. The ratio of the force constants, $k_{r} / k_{\theta}$, can also be varied to transform the system from one which is more like atomic material (length dominated) to a foam (angle dominated). In addition, the possibility of using the $\mathrm{ReB}$ angle potential is an elegant way to maintain ring convexity, providing that any nonconvexity introduced by the bond switch is resolved before the geometry optimization takes place. Finally a major advantage of the bond switching algorithm is that a local geometry optimization can be employed such that only the atoms in the immediate vicinity of the switching move need to be minimized to obtain an accurate structure [64], which in our work included all atoms within five coordination shells.

Unless otherwise stated, the results in this work were obtained for 1024 ring systems for $\langle k\rangle=3,4$ and 1152 ring systems for $\langle k\rangle=5$. Each was thermalized with $2 \times 10^{5}$ random moves and annealed over a further $4 \times 10^{6}$ moves. For each system 100 simulations were run starting from different random seeds.

\section{B. Triangle raft}

The concept of using triangle rafts to represent glasses dates back to Zachariasen [65] and was used to construct some of the first models [66] of amorphous materials. The principle is to build a network in a sequential manner by adding rings consisting of triangular subunits. Unlike bond switching, this method is not constrained by a periodic boundary and mimics an "organic" growth mechanism. An improved version of this method has recently been implemented computationally [67], such that rings are added with probabilities based on their relative energetics as opposed to purely randomly. Once a site to add a new ring has been identified, rings of different sizes are trialled, geometry optimized, and accepted with probability:

$$
P_{k}=\frac{e^{-\Delta U_{k} / T}}{\sum_{k} e^{-\Delta U_{k} / T}},
$$

where $\Delta U_{k}$ is the energy change on adding a ring of size $k$ and $T$ is a temperature parameter. This temperature allows the degree of disorder to be controlled, which is not possible with the original algorithm. Samples generated in this way have proved a good proxy to amorphous materials such as silica bilayers. In this work some 27500 networks consisting of 1000 rings were constructed across a range of temperatures to sample all available ring statistics. 


\section{Hard-disk Monte Carlo}

Hard-disk Monte Carlo has been extensively studied [68] and simple hard-disk Monte Carlo simulations were performed here to supplement experimental colloid data. These involved placing $N=1000$ disks in a square cell with periodic boundary conditions at a given packing fraction in the range $\phi=0.0 \rightarrow 0.77$ (the loose random packing limit), and then performing Monte Carlo cycles of $N$ random displacement moves. Each simulation consisted of $10^{5}$ equilibration cycles and $10^{5}$ production cycles, with sampling every 10 production cycles. For each packing fraction 10 calculations were run using a different random seed, the results compiled, and a Voronoi analysis was performed for each configuration to generate a system of tessellating rings [69].

\section{EXPERIMENTAL METHODS}

\section{A. Colloid monolayers}

The quasi-2D colloidal system has been shown to be an excellent model for hard disks, as has been previously discussed [70]. It consists of particles with a diameter of $\sim 2.79 \mu \mathrm{m}$ dispersed in a water-ethanol mixture and confined by gravity to form a monolayer on the base of a glass sample cell. Out-of-plane fluctuations are quantified by the gravitational height of the particles, which is a very small percentage of their diameter, and as such the system is structurally two dimensional. Monolayers are considered at a variety of packing fractions from $\phi=0.29$ to $\phi=0.66$, which correspond to the fluid phase of the system [70]. Samples are imaged using an inverted bright-field microscope and particle coordinates obtained using standard particle tracking routines. Quantities discussed here are calculated from 100 frames of the system, with the time between frames around $10 \mathrm{~s}$. At the highest packing fractions considered, the area of the system imaged contains around 3000 particles. As the system is inherently aperiodic, after Voronoi analysis [69] the cells on the image boundary are neglected.

\section{B. Geopolitical regions}

Common real-world examples of two-dimensional tilings are geopolitical maps. Physicists have previously studied the regions of France and Ireland and noted the similarity in their properties to materials [13,71]. Therefore, we have continued this tradition by analyzing five further maps: the communes of Switzerland, the parishes and Westminster constituencies of Great Britain, and the socio-economic regions of the European Union (EU) and European Free Trade Association (EFTA) (including both current and candidate countries at the time of writing). The shapefiles for these regions are available from the relevant institutions [72].

In geopolitical tilings an administrative region on the map is defined by its boundary. Regions are said to be neighbors if they share at least one point anywhere along the boundary, such that the region size is then given by the number of neighbors. Therefore, vertices are formed where three regions share a boundary and edges where two regions share a boundary. Analysis of these networks is slightly complicated by the possible presence of defects. Defects arise when regions have $k<3$ neighbors, either as a result of small imperfections in
TABLE IV. Summary of map network analysis. The number of total and interior regions (without defects) are given for each map. The interior regions were then used to calculate the network properties.

\begin{tabular}{lcccccc}
\hline \hline Region & Total & Interior & $\langle k\rangle$ & $p_{6}$ & $\mu_{2}$ & $r$ \\
\hline CH & 2379 & 2051 & 5.914 & 0.206 & 3.825 & -0.151 \\
GB (parish) & 11663 & 10778 & 6.005 & 0.241 & 3.028 & -0.163 \\
GB (const.) & 654 & 455 & 5.930 & 0.251 & 3.019 & -0.110 \\
EU and EFTA & 387 & 145 & 5.897 & 0.283 & 1.913 & -0.215 \\
EU and EFTA & 1617 & 972 & 5.910 & 0.271 & 2.531 & -0.161 \\
\hline
\end{tabular}

the boundary data or from legitimate region arrangements. For example, if $k=0$, then the region is an island; if $k=1$, then a region is fully inscribed within another (usually indicative of a large urban area); and if $k=2$, then a region sits on a ring edge. As these structures are primarily for illustrative purposes, these defects can be simply discounted for the purposes of the network analysis. A summary of the results from these geopolitical tilings is given in Table IV, while visualizations are provided in Figs. 1 and 5.

\section{Atomic materials}

In addition to samples generated as part of this work, data were also supplemented with published experimental coordinates of amorphous silica and graphene [1,2,24]. Atomic networks were generated from these coordinates by connecting atoms within a given cutoff. For each sample, defects were identified from the presence of under-coordinated atoms, arising largely from the sample perimeter or from holes in the center. As for the geopolitical regions above, such defects can be removed before performing network analysis.
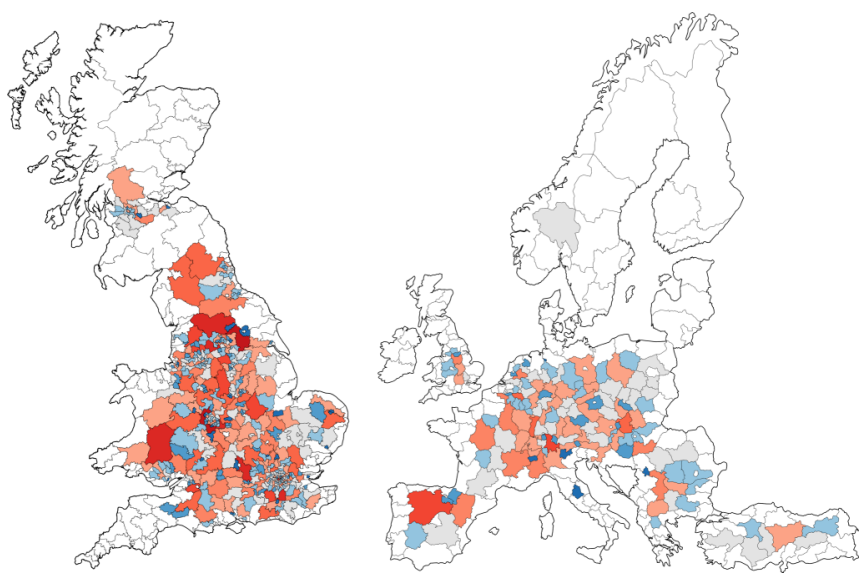

FIG. 5. Two example geopolitical partitions used in this work are the Westminster constituencies of Great Britain and the socioeconomic regions of the EU (current and candidate countries) and EFTA [72]. 


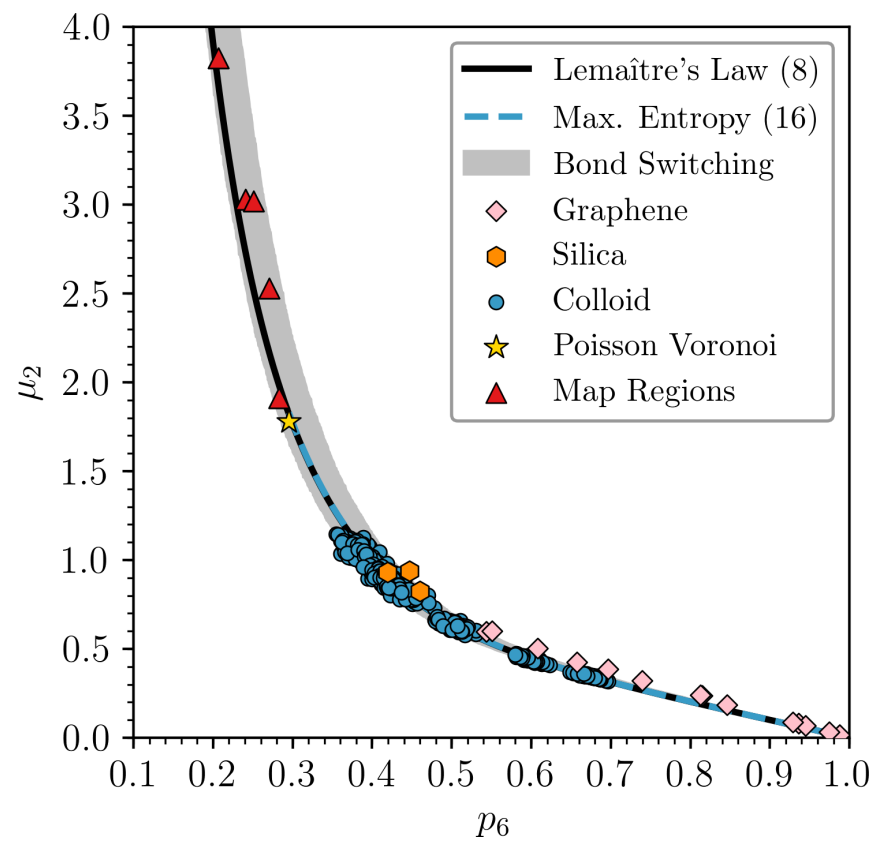

FIG. 6. The maximum entropy ring distribution can be summarized in Lemaittre's law, which relates the proportion of hexagons, $p_{6}$, against the variance, $\mu_{2}$ (black line). Comparisons are given to bond switching simulations of two-dimensional materials (gray area representing two standard deviations from the mean), amorphous graphene (pink diamonds) [24], silica bilayers (orange hexagons) [1,2], experimental colloidal monolayers (blue circles), the Poisson Voronoi diagram (yellow star), and maps of geopolitical regions (red triangles) [72]. Lemaître's law is regenerated using maximum entropy methods with the assortativity as a constraint, shown here using $p_{6}$ and $r$ values from hard-disk simulations (blue dashed line).

\section{RESULTS}

\section{A. Degree distribution}

The degree distributions of physical networks are discussed in terms of Lemaitre's law with the distribution variance, $\mu_{2}$, plotted against the proportion of rings of mean size. Figure 6 presents these data for a range of 3-coordinate systems comprising experimental, computational, and theoretical. There are many things to note, but primarily we see that regardless of the nature of the underlying system, all data fit very well with the maximum entropy solution provided by Lemaitre's law. While Lemaître's law highlights the similarities between these systems, it is also important to examine some of their differences. For example, what determines where a system sits on the Lemaître curve, i.e., what controls the number of hexagons? For materials this is based on energeticsthe strain associated with bond and angle distortions. For instance, experimentally silica bilayers have more diverse ring statistics than graphene due to the reduction in ring strain due to the presence of oxygen linkages [2]. Even for the graphene samples which have been modified by an electron beam (pink diamonds), the disorder does not approach that of the silica glasses (orange hexagons). For the colloid systems (blue circles), however, the rings are formed from the Voronoi tessellation, with no intrinsic cost to distortions and instead it is the packing fraction, $\phi$, which determines $p_{6}$. The limit $\phi \rightarrow 0$ achieves the Poisson Voronoi ring distribution (yellow star) [73], with a lower bound of $p_{6} \approx 0.295$. For the administrative geopolitical regions (red triangles), there is no energy cost for rings, regardless of shape, convexity, or separation, and so we find these points in the low- $p_{6}$, high-entropy portion of Lemaître's curve.

On the other hand, using a flexible computational method allows access to the entire range of $\mu_{2}$ values, where the level of disorder is controlled by the Monte Carlo "temperature" parameter. The results from bond switching highlight the typical dispersion that can be expected within Lemaître's law, with the gray shaded region indicating the bounds of $\mu_{2}$ within two standard deviations of the mean. Finally, we see that using Eq. (16) with the $p_{6}$ and $r$ values from hard-disk Monte Carlo (blue dashed line) reproduces the results from Lemaître's law without the need for the empirical constraint. The calculation of this line is explained at the end of Sec. V B.

The effect on the maximum entropy solutions can also be explored for different atomic coordination environments and constraints. Figure 7 gives two such examples where ring convexity is enforced by using the ReB potential. Figure 7(a) gives results for a purely 3 -coordinate system, $x_{3}=1$, while Fig. 7(b) gives results for a purely 4-coordinate system, $x_{4}=$ 1. The maximum solution each case is again given by Eq. (8), with $\langle k\rangle=6,4$, respectively. The value of $\mu_{2}$ is very similar for $\langle k\rangle=4$ and $\langle k\rangle=6$ above $p_{\langle k\rangle} \approx 0.5$. This is because in this region rings of sizes $k=\langle k\rangle$ and $k=\langle k\rangle \pm 1$ dominate the distribution and so $\mu_{2} \approx 1-p_{\langle k\rangle}$. However, as the value of $p_{\langle k\rangle}$ is reduced further, the two maximum entropy curves begin to diverge as the $k=\langle k\rangle-2$ ring becomes accessible only to the 3-coordinate system, which in turn facilitates the presence of higher-order rings. In Figs. 7(a) and 7(b), the results from bond switching both begin to deviate from the analytical results of Lemaitre's law at low $p_{\langle k\rangle}$. The origins of this deviation can be traced back to the fact that if ring convexity is strictly maintained, it becomes increasingly difficult to accommodate the very large rings required to achieve large $\mu_{2}$ values.

\section{B. Assortativity}

The ring correlations as measured by the assortativity are given for all 3-coordinate systems in Fig. 8(a). We find that all these 3-coordinate networks are disassortative and lie in the region $-0.35<r<-0.10$ and that curves display a similar characteristic shape. The experimental colloid samples are well described by the hard-disk model (blue circles and blue central line), with $p_{6} \approx 0.84$ corresponding to packing fractions above the freezing transition limit $(\phi \approx 0.70)$ [74]. The curves generated from bond switching (gray lines) and triangle rafts (orange lowest line) display different assortativities which depend on the balance of the length- and angle-driven forces. The driving force for the hard-disk model is purely entropic, whereas for the other methods there is also a complex energy landscape, which may favor specific assortativities [57] and which can be "tuned" by altering the balance of the interactions. For example, the bond switching results show the effect of varying this balance with $k_{r} / k_{\theta}=$ $16,4,1,1 / 4$ (black to light gray lines), leading to a shifting in the assortativity curves. We note that this is supported by the experimental results from amorphous graphene (pink 

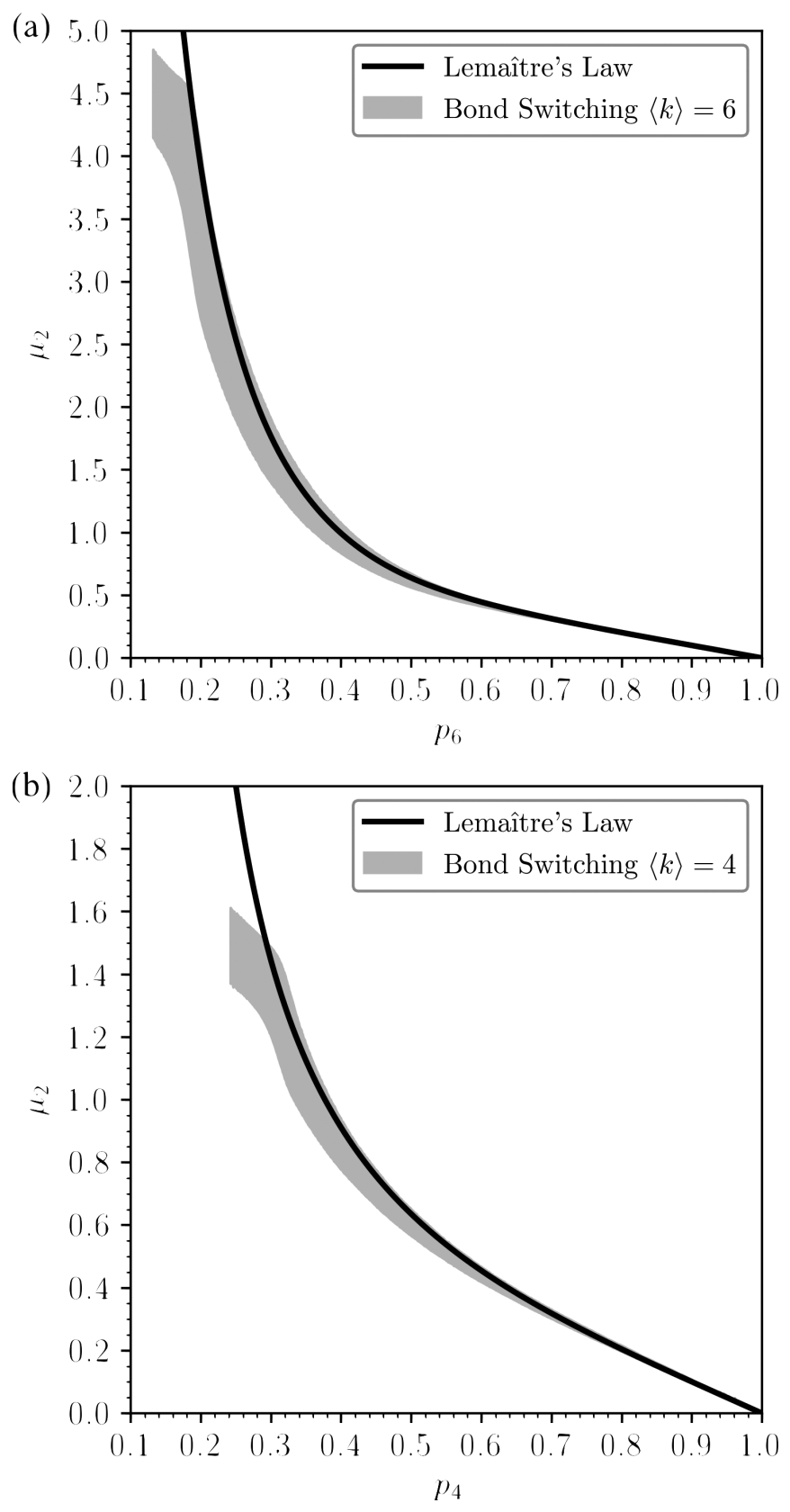

FIG. 7. Comparison of Lemaître's law (black line) for purely 3- and 4-coordinate systems, panels (a) and (b), respectively. Bond switching results using the ReB potential are also shown (gray area representing two standard deviations from the mean).

diamonds), which lie in the between the two curves with the largest bond length to angle force constant ratio, as would be intuitively expected for atomic systems and from empirical potential models [26].

For all the systems we note that there are different regimes, with the high- $p_{6}$ limit corresponding to configurations best described as crystalline with defects rather than truly amorphous as in the low- $p_{6}$ limit-with the two often being linked by a phase transition. The high- $p_{6}$ limit can be rationalized by considering the frequency of common defect types at infinite dilution in a hexagonal lattice [75,76] [Figs. 8(b)-8(e)]. These can be calculated by considering the explicit edge joint

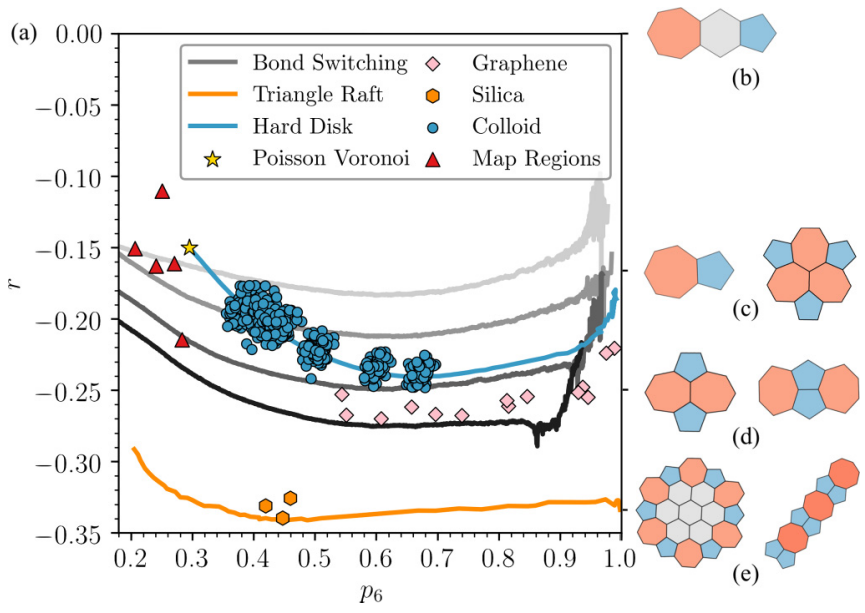

FIG. 8. (a) Variation in assortativity against $p_{6}$ for a range of 3-coordinate systems comprising experimental and simulation data. Simulation data: bond switching (gray lines, darker indicating greater $k_{r} / k_{\theta}$ ), triangle raft (orange lowest line), hard-disk Monte Carlo (blue central line). Experimental data: amorphous graphene (pink diamonds) [24], silica bilayers (orange hexagons) [1,2], experimental colloidal monolayers (blue circles), and maps of geopolitical regions (red triangles) [72]. The yellow star indicates the Poisson Voronoi limit. Panels (b)-(e) show common defects found in crystalline systems, placed at their limiting assortativity value (b) isolated pair, $r=$ 0.0 ; (c) adjacent pair, cluster, $r=-0.1 \dot{6}$; (d) Stone-Wales, mitosis, $r=-0.25$; (e) 5-7 chain (flower defect), 5-8 chain, $r=-0 . \dot{3}$.

probability distribution for a specific defect. For example, for the Stone-Wales defect [Fig. 8(d)], we see that each 5-ring has two 7-ring neighbors, and each 7-ring two 5-ring and one 7-ring neighbors such that:

$$
\boldsymbol{e}=\left[\begin{array}{ccc}
5 & 6 & 7 \\
0 & 3 \epsilon & 2 \epsilon \\
3 \epsilon & 1-19 \epsilon & 4 \epsilon \\
2 \epsilon & 4 \epsilon & \epsilon
\end{array}\right] \begin{gathered}
5 \\
6 \\
7
\end{gathered}
$$

where $\epsilon=\left(1-p_{6}\right) / 12$. From here it is simple to evaluate the dilute $p_{6}$ limit as $\lim _{\epsilon \rightarrow 0} r=\frac{1}{4}$.

This helps to rationalize the high- $p_{6}$ disassortative behavior for these 3-coordinate systems. For hard disks as $p_{6} \rightarrow 1$ the adjacent pair appears to be dominant, whereas for bond switching and triangle rafts the potential model determines the balance of defect types. For bond switching the standard deviation is large as each sample contains a single defect corresponding to one of the low-energy forms. By visual inspection, increasing the length relative to the angle driving force preferences chainlike structures over isolated defects. Similarly, the rigidity of the triangle units in the triangle raft method leads to a very tight length distribution which encourages the formation of defects such as Fig. 8(e). As $p_{6}$ decreases more defects are introduced and the system becomes truly amorphous. Again we posit that as the harddisk model has no energetic term, it is able to incorporate less correlated defects, and in the low packing fraction the hard-disk model provides an estimate for the Poisson Voronoi limit of $r \approx-0.15$.

Again the effects of coordination environment and potential model on assortativity in complex networks can be 


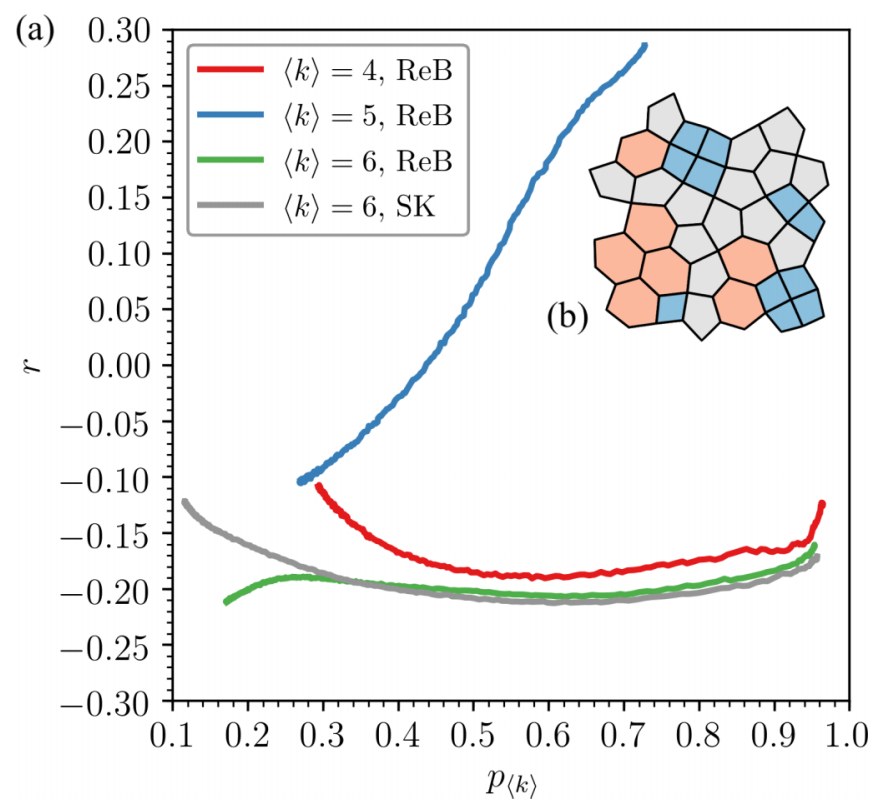

FIG. 9. Panel (a) shows the variation in assortativity, with ring statistics for 3-coordinate (green, gray bottom lines), 4-coordinate (red middle line), and mixed 3/4-coordination systems (blue top line) using the SK and ReB potentials as indicated. Panel (b) gives a fragment of a mixed coordination lattice displaying clustering of rings of similar size.

demonstrated using bond switching. Figure 9(a) shows such a comparison, where the assortativity is plotted against the primary ring size for different coordination environments, averaged by Monte Carlo temperature. The effect of imposing a hard constraint on ring convexity can be seen through the two curves corresponding to $\langle k\rangle=6$. These curves show very similar behavior for $p_{6} \gtrsim 0.3$, below which there is increasing deviation. This is as expected given the violation of ring convexity will only occur for very large rings at high temperatures, which can undergo deformation to reduce bond angle strain. This allows larger rings to pack next to each other, reducing the disassortativity. The behavior of the pure 4-coordinate system, $\langle k\rangle=4$, is qualitatively the same as for the 3-coordinate network, and indeed all the defects in Fig. 8 have analogs in 4-coordinate networks. The network of greater interest is that with mixed 3- and 4-coordinate vertices, corresponding to $\langle k\rangle=5$. In this case we see fundamentally different properties as these networks are assortative at high $p_{5}$, in contrast to limiting pure coordination cases. This assortative mixing is readily explainable through energetic considerations. The hexagonal and square tilings are strainless and so the disruptive effects of any defect rings is minimized when such rings are adjacent. Unlike the hexagonal and square lattices, the Cairo lattice is not strainless, due to a distortion in one of the edge lengths in the pentagonal tiles. Therefore, any 4- or 6-ring defects experience a driving force to cluster into the low-energy regular tilings. In effect the lattice demixes into Cairo, square, and hexagonal regions [as in Fig. 9(b)], which we identify as inherently assortative behavior. It is for this same reason that the limit of $p_{5} \rightarrow 1$ cannot be reached, as the minimum energy lattice will be a mixture of the square,

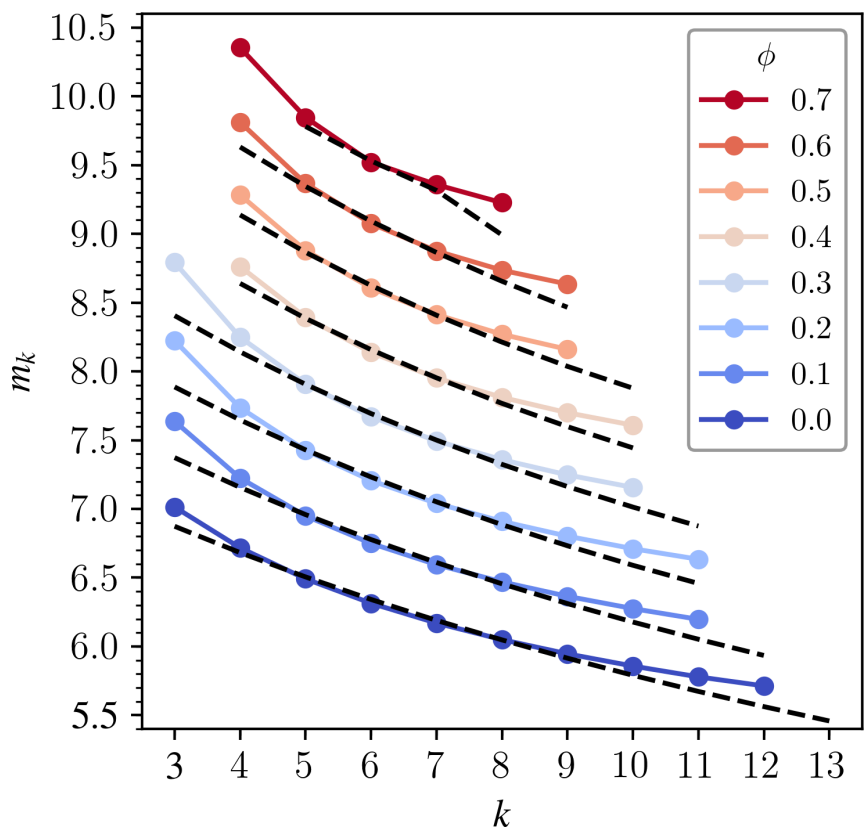

FIG. 10. Mean ring size of hard-disk simulations at different packing fractions (full lines) compared to results from maximum entropy (dashed lines). In both cases only ring sizes with $p_{k}>10^{-4}$ are displayed and results are offset by 0.5 along the abscissa for clarity.

hexagonal and Cairo lattices, the exact proportion of which will depend on the potential model.

Finally, we assess the accuracy of our extension to Lemaître's maximum entropy method in Eq. (16). Calculation of the maximum entropy joint degree distribution requires two parameters, $p_{6}$ and $r$, but the resulting distribution contains all the information required to calculate ring statistics, $p_{k}$, and the mean ring size about each ring, $m_{k}$. This has been performed using the parameters of $p_{6}$ and $r$ from hard-disk simulations. As demonstrated in Fig. 6, the ring statistics calculated in this way regenerate those from Lemaitre's law. In addition, plots of the mean ring sizes for selected packing fractions are given in Fig. 10. While the fit is not perfect, this method does provide a close approximation to the hard-disk results, particularly in the vicinity of $k=\langle k\rangle$. The results are especially good in the context that only two variables are required in $p_{6}$ and $r$ to generate the distributions.

\section{Extension to spherical topology}

As an illustration of the generalizability of the methods described in this work, we present results for two-dimensional networks in spherical topology. Such systems are also of experimental interest, as we now have access to "nonclassical" fullerenes [77-80], metal-organic nanocages [81,82], as well as curved froths [83]. We investigated one such fullerene: a 92-ring 3-coordinate fullerene consisting of 5-, 6-, and 7rings. Possible configurations were again generated via bond switching, starting from the lattice depicted in Fig. 3(e). Here $10^{6}$ total configurations were sampled from 100 different simulations, with $k_{r} / k_{\theta}=4$. 


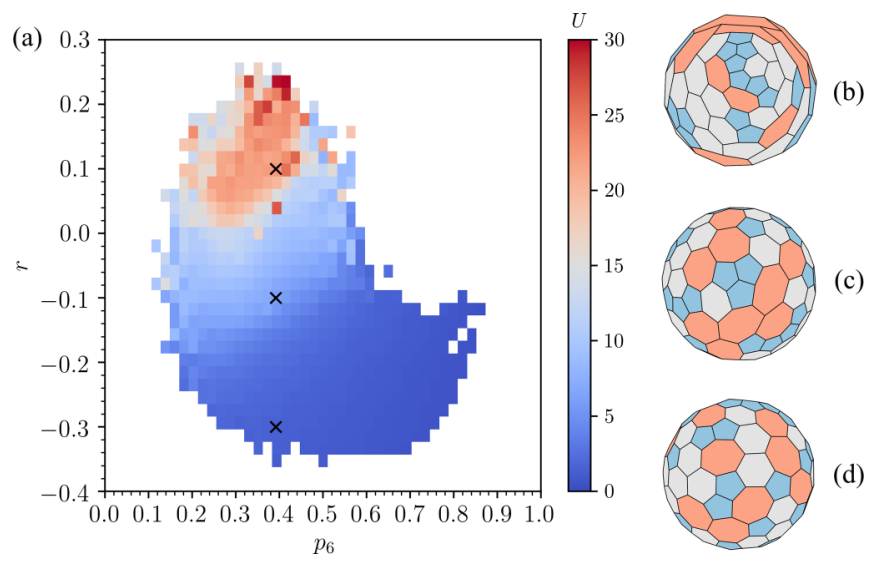

FIG. 11. Panel (a) gives a map of fullerene stability as a function of ring statistics and assortativity. Potential energy increases as more pentagons and heptagons are accommodated but is also strongly related to their arrangement as shown by the value of the assortativity, $r$. Panels (b)-(d) give three example fullerenes with the same $p_{6}=$ $36 / 92$ but different assortativities of $r \approx 0.1,-0.1,-0.3$, respectively, and as highlighted by the crosses in panel (a).

Results of the network properties averaged across configurations are given in Fig. 11(a), colored by potential energy. In this plot the value of $p_{6}$ is discretized, due to the the small and well-defined number of rings, and cannot exceed the upper limit imposed by the 12-pentagon rule, whereas the assortativity is averaged. As expected, the energy of the fullerenes increases with the increasing diversity in the ring statistics, as more pentagons and heptagons are accommodated. However, we also see that the arrangement of the rings, as measured by the assortativity, is also very important in determining the stability of the networks. To emphasize this, three example configurations are provided in Figs. 11(b)-11(d). These amorphous fullerenes have the same $p_{6}$ value (and therefore $p_{5}$ and $p_{7}$ ) but very different strain energies. In Fig. 11(d) we see defects similar to the common motifs as in Fig. 8, which we associate with being low energy. The increased clustering of similar-sized rings in Figs. 11(b) and 11(c) leads to increasingly irregular ring geometries that generate high levels of strain. As previously noted with planar networks, systems which are disassortative are energetically favored. Although this is a simple consequence of the mechanical properties of the system, neglecting any electronic contributions, such is the difference in stability that we would expect disassortative fullerenes of this type to be more prevalent in nature.

\section{CONCLUSION}

In summary, we have thoroughly examined the network properties of a wide range of naturally occurring two-dimensional systems; spanning varying coordination environments, potential models, and topologies. Data have been collected from a range of experimental sources, and we have further developed the theoretical bond switching method to aid the study of these diverse systems computationally. We have analyzed these data with rigorous metrics from network science, with the aim of highlighting the study of real-world physical systems as an important and interesting addition to the wider field. In particular, these networks display unique constraints as a result of their underlying physics. We have shown that their mean node degree is fixed and the node degree distribution is well defined, following Lemaitre's law. In addition, we have investigated the network assortativity, arguing its preferability over the previous empirical measure known as the Aboav-Weaire law. Although the assortativity has been shown to be a function of the potential model for a system and the limits of the assortativity linked to the occurrence of well-known physical motifs; most physical networks show a very similar overall level of disassortativity, as experienced in nature. An exception to this rule has also been found, where variable-coordination systems can demix to exhibit assortative behavior.

In this work we have demonstrated how network science is applicable to understanding and analyzing generic systems in physics but also how physical systems form a key and underexplored area of network science. Going forward, additional work can be done to more strongly link these two subjects for mutual benefit. For example, there are still questions to be answered from this work, such as how network properties such as the assortativity are explicitly related to the physics of the underlying system and whether this information can be utilized experimentally, for example, to control and effectively quantify the pore size in materials. More broadly, there are natural extensions to highly disordered networks which are far less constrained in terms of coordination environments and potential model, as found often in biology, as well as to three-dimensional networks.

\section{ACKNOWLEDGMENTS}

We are grateful for support from the EPSRC Centre for Doctoral Training in Theory and Modelling in Chemical Sciences (TMCS), under Grant No. EP/L015722/1 and to the UK Materials and Molecular Modelling Hub for computational resources, which is partially funded by EPSRC (EP/P020194/1). This paper conforms to the RCUK data management requirements.
[1] P. Y. Huang, S. Kurasch, A. Srivastava, V. Skakalova, J. Kotakoski, A. V. Krasheninnikov, R. Hovden, Q. Mao, J. C. Meyer, J. H. Smet et al., Nano Lett. 12, 1081 (2012).

[2] C. Büchner and M. Heyde, Prog. Surf. Sci. 92, 341 (2017).

[3] A. W. Robertson, G.-D. Lee, K. He, E. Yoon, A. I. Kirkland, and J. H. Warner, Nano Lett. 14, 3972 (2014).
[4] J. C. Earnshaw and D. J. Robinson, Phys. Rev. Lett. 72, 3682 (1994).

[5] C. Allain and L. Limat, Phys. Rev. Lett. 74, 2981 (1995).

[6] M. Durand, J. Käfer, C. Quilliet, S. Cox, S. A. Talebi, and F. Graner, Phys. Rev. Lett. 107, 168304 (2011). 
[7] M. Tong, K. Cole, P. R. Brito-Parada, S. Neethling, and J. J. Cilliers, Langmuir 33, 3839 (2017).

[8] M. C. Gibson, A. B. Patel, R. Nagpal, and N. Perrimon, Nature 442, 1038 (2006).

[9] R. Carter, Y. E. Sánchez-Corrales, M. Hartley, V. A. Grieneisen, and A. F. M. Marée, Development 144, 4386 (2017).

[10] L. Goehring and S. W. Morris, Phys. Today 67(11), 39 (2014).

[11] D. Brutin, B. Sobac, B. Loquet, and J. Sampol, J. Fluid Mech. 667, 85 (2011).

[12] F. Glassmeier and G. Feingold, Proc. Natl. Acad. Sci. USA 114, 10578 (2017).

[13] G. Le Caër and R. Delannay, J. Phys. I Fr. 3, 1777 (1993).

[14] G. Schliecker and S. Klapp, Europhys. Lett. 48, 122 (1999).

[15] W. T. Gibson, J. H. Veldhuis, B. Rubinstein, H. N. Cartwright, N. Perrimon, G. W. Brodland, R. Nagpal, and M. C. Gibson, Cell 144, 427 (2011).

[16] M. K. Ladan, P. Ziherl, and A. Šiber, Phys. Rev. E 100, 012410 (2019).

[17] J. Kotakoski, A. V. Krasheninnikov, U. Kaiser, and J. C. Meyer, Phys. Rev. Lett. 106, 105505 (2011).

[18] A. Malashevich, S. Ismail-Beigi, and E. I. Altman, J. Phys. Chem. C 120, 26770 (2016).

[19] D. Weaire and N. Rivier, Contemp. Phys. 50, 199 (2009).

[20] J. C. Flores, Soft Matter 13, 1352 (2017).

[21] A.-L. Barabási, Nat. Phys. 8, 14 (2012).

[22] S. Boccaletti, V. Latora, Y. Moreno, M. Chavez, and D.-U. Hwang, Phys. Rep. 424, 175 (2006).

[23] S. H. Strogatz, Nature 410, 268 (2001).

[24] F. R. Eder, J. Kotakoski, U. Kaiser, and J. C. Meyer, Sci. Rep. 4, 4060 (2014).

[25] G. L. Caër and R. Delannay, J. Phys. A 26, 3931 (1993).

[26] A. Kumar, M. Wilson, and M. F. Thorpe, J. Phys.: Condens. Matter 24, 485003 (2012).

[27] E. Ressouche, V. Simonet, B. Canals, M. Gospodinov, and V. Skumryev, Phys. Rev. Lett. 103, 267204 (2009).

[28] P. W. Fowler, T. Heine, D. E. Manolopoulos, D. Mitchell, G. Orlandi, R. Schmidt, G. Seifert, and F. Zerbetto, J. Phys. Chem. 100, 6984 (1996).

[29] A. Kumar, D. Sherrington, M. Wilson, and M. F. Thorpe, J. Phys.: Condens. Matter 26, 395401 (2014).

[30] J. Shackelford and B. D. Brown, J. Non. Cryst. Solids 44, 379 (1981).

[31] R. Delannay and G. Le Caër, Phys. Rev. Lett. 73, 1553 (1994).

[32] P. Cerisier, S. Rahal, and N. Rivier, Phys. Rev. E 54, 5086 (1996).

[33] M. P. Miklius and S. Hilgenfeldt, Phys. Rev. Lett. 108, 015502 (2012).

[34] A. Gervois, J. P. Troadec, and J. Lemaitre, J. Phys. A 25, 6169 (1992).

[35] F. T. Lewis, Anat. Rec. 38, 341 (1928).

[36] M. A. Fortes, J. Phys. A 28, 1055 (1995).

[37] S. Kim, M. Cai, and S. Hilgenfeldt, New J. Phys. 16, 015024 (2014).

[38] S. N. Chiu, Mater. Charact. 34, 149 (1995).

[39] D. A. Aboav, Metallography 3, 383 (1970).

[40] D. Weaire, Metallography 7, 157 (1974).

[41] S. Le Roux and F. Rezai-Aria, J. Phys. D 46, 295301 (2013).

[42] P. K. Roy, M. Heyde, and A. Heuer, Phys. Chem. Chem. Phys. 20, 14725 (2018).
[43] A. Noever, Colloids Surf. 62, 243 (1992).

[44] J. C. M. Mombach, R. M. C. de Almeida, and J. R. Iglesias, Phys. Rev. E 47, 3712 (1993).

[45] P. Pedro, J. Saraiva, L. Bandeira, and J. Antunes, Planet. Space Sci. 56, 1919 (2008).

[46] H. J. Hilhorst, J. Phys. A 39, 7227 (2006).

[47] S. Kumar, S. K. Kurtz, and D. Weaire, Philos. Mag. B 69, 431 (1994).

[48] M. E. J. Newman, Phys. Rev. Lett. 89, 208701 (2002).

[49] R. Noldus and P. V. Mieghem, J. Complex Networks 3, 507 (2015).

[50] A. Chremos and P. J. Camp, Phys. Rev. E 76, 056108 (2007).

[51] N. Litvak and R. van der Hofstad, Phys. Rev. E 87, 022801 (2013).

[52] J. K. Mason, R. Ehrenborg, and E. A. Lazar, J. Phys. A 45, 065001 (2012).

[53] F. Wooten, K. Winer, and D. Weaire, Phys. Rev. Lett. 54, 1392 (1985).

[54] V. Meunier, A. G. Souza Filho, E. B. Barros, and M. S. Dresselhaus, Rev. Mod. Phys. 88, 025005 (2016).

[55] J. M. Greneche and J. M. D. Coey, J. Phys. Fr. 51, 231 (1990).

[56] N. Metropolis, A. W. Rosenbluth, M. N. Rosenbluth, A. H. Teller, and E. Teller, J. Chem. Phys. 21, 1087 (1953).

[57] D. Ormrod Morley and M. Wilson, J. Phys.: Condens. Matter 30, 50LT02 (2018).

[58] F. Bamer, F. Ebrahem, and B. Markert, Materialia 9, 100556 (2020).

[59] P. N. Keating, Phys. Rev. 145, 637 (1966).

[60] G. T. Barkema and N. Mousseau, Phys. Rev. B 62, 4985 (2000).

[61] S. K. Jain and G. T. Barkema, PCCP 20, 16966 (2018).

[62] S. von Alfthan, A. Kuronen, and K. Kaski, Phys. Rev. B 68, 073203 (2003).

[63] M. Bulacu, N. Goga, W. Zhao, G. Rossi, L. Monticelli, X. Periole, D. P. Tieleman, and S. J. Marrink, J. Chem. Theory Comput. 9, 3282 (2013).

[64] N. Mousseau and G. T. Barkema, Curr. Opin. Solid State Mater. Sci. 5, 497 (2001).

[65] W. Zachariasen, J. Am. Chem. Soc. 54, 3841 (1932).

[66] J. F. Shackelford, J. Non. Cryst. Solids 49, 19 (1982).

[67] D. Ormrod Morley and M. Wilson, Mol. Phys. 117, 3148 (2019).

[68] M. Isobe, Mol. Simul. 42, 1317 (2016).

[69] C. H. Rycroft, Chaos 19, 041111 (2009).

[70] A. L. Thorneywork, J. L. Abbott, D. G. A. L. Aarts, and R. P. A. Dullens, Phys. Rev. Lett. 118, 158001 (2017).

[71] A. Okabe, B. Boots, and K. Sugihara, Spatial Tessellations: Concepts and Applications of Voronoi Diagrams (Wiley, New York, 1992).

[72] Ordnance Survey, Boundary-Line Data, (2018); Federal Office of Topography, swissBOUNDARIES3D (2019); Eurostat, NUTS Geodata: EuroGeographics for the Administrative Boundaries (2016).

[73] M. Tanemura, Forma 18, 221 (2003).

[74] E. P. Bernard and W. Krauth, Phys. Rev. Lett. 107, 155704 (2011).

[75] A. H. Marcus and S. A. Rice, Phys. Rev. E 55, 637 (1997). 
[76] T. Björkman, S. Kurasch, O. Lehtinen, J. Kotakoski, O. V. Yazyev, A. Srivastava, V. Skakalova, J. H. Smet, U. Kaiser, and A. V. Krasheninnikov, Sci. Rep. 3, 3482 (2013).

[77] H.-R. Tian, M.-M. Chen, K. Wang, Z.-C. Chen, C.-Y. Fu, Q. Zhang, S.-H. Li, S.-L. Deng, Y.-R. Yao, S.-Y. Xie et al., J. Am. Chem. Soc. 141, 6651 (2019).

[78] R. Guan, F. Jin, S. Yang, N. B. Tamm, and S. I. Troyanov, Inorg. Chem. 58, 5393 (2019).

[79] V. A. Brotsman, D. V. Ignateva, and S. I. Troyanov, Chem. Asian J. 12, 2379 (2017).
[80] V. A. Brotsman, N. B. Tamm, V. Y. Markov, I. N. Ioffe, A. A. Goryunkov, E. Kemnitz, and S. I. Troyanov, Inorg. Chem. 57, 8325 (2018).

[81] D. Fujita, Y. Ueda, S. Sato, N. Mizuno, T. Kumasaka, and M. Fujita, Nature 540, 563 (2016).

[82] Z. Wang, H.-F. Su, Y.-Z. Tan, S. Schein, S.-C. Lin, W. Liu, S.-A. Wang, W.-G. Wang, C.-H. Tung, D. Sun et al., Proc. Natl. Acad. Sci. USA 114, 12132 (2017).

[83] A. E. Roth, C. D. Jones, and D. J. Durian, Phys. Rev. E 86, 021402 (2012). 